\title{
STUDY OF OVARIAN LESIONS IN CHILDREN
}

\section{K. Nagarjuna, ${ }^{1}$ S. Srinivas ${ }^{2}$, K. Ramesh Reddy ${ }^{3}$, K. Lavanya ${ }^{4}$, Inugala Anusiri ${ }^{5}$}

${ }^{1}$ Associate Professor, Department of Paediatric Surgery, Niloufer Hospital, Osmania Medical College, Hyderabad. ${ }^{2}$ Assistant Professor, Department of Paediatric Surgery, Niloufer Hospital, Osmania Medical College, Hyderabad. ${ }^{3}$ Professor \& HOD, Department of Paediatric Surgery, Niloufer Hospital, Osmania Medical College, Hyderabad. ${ }^{4}$ Assistant Professor, Department of Paediatric Surgery, Niloufer Hospital, Osmania Medical College, Hyderabad. 5 Post Graduate, Department of Paediatric Surgery, Niloufer Hospital, Osmania Medical College, Hyderabad.

ABSTRACT: AIM: To study ovarian lesions in paediatric patients.

MATERIALS AND METHODS: All cases of ovarian lesions presenting to Niloufer Hospital during the study period spanning 7 years were included in the study. All cases were clinically examined and evaluated with serum markers like $\alpha$-fetoprotein and $\beta$-HCG, ultrasonography of abdomen and pelvis and contrast enhanced computed tomography of abdomen and pelvis. The cases were managed either conservatively or surgically depending on the diagnosis.

RESULTS: A total of 36 cases of ovarian lesions presented to Niloufer Hospital during the study period. The age of the patients ranged from newborn to 14 years. The most common presenting symptom was an abdominal or pelvic mass. The most common ultrasound character of the non-neoplastic ovarian lesion was cystic whereas that of a neoplastic ovarian lesion was complex or mixed. Non neoplastic lesions were present in 22 patients. Neoplastic lesions were seen in 14 patients.

CONCLUSION: Physiological or functional ovarian cysts are the commonest ovarian lesions seen in the paediatric age group. Ultrasonogram is accurate and very reliable in distinguishing neoplastic from non-neoplastic and malignant from benign ovarian lesions. Non-neoplastic lesions can be managed conservatively in about $40 \%$ of cases. Neoplastic lesions in children require a less radical approach in order to preserve ovarian function.

KEYWORDS: Ovarian Lesion, Teratoma.

HOW TO CITE THIS ARTICLE: K. Nagarjuna, S. Srinivas, K. Ramesh Reddy, K. Lavanya, Inugala Anusiri. "Study of Ovarian Lesions in Children". Journal of Evolution of Medical and Dental Sciences 2015; Vol. 4, Issue 90, November 09; Page: 15534-15536, DOI: $10.14260 /$ jemds/2015/2225.

INTRODUCTION: Ovarian lesions are relatively rare in paediatric age group. ${ }^{1}$ and those requiring hospital admissions are even more uncommon. Nevertheless these lesions need to be considered in the differential diagnosis in all girls with abdominal pain, abdominal mass or precocious puberty. ${ }^{2}$ Ovarian lesions in paediatrics include nonneoplastic and neoplastic masses. Non-neoplastic masses include functional cysts and neoplastic lesions include benign and malignant tumors of the ovary. Ovarian tumors make upto $1 \%$ of all childhood malignancies. ${ }^{1}$ Removal of ovary may have significant impact on future fertility prospects of a young patient. ${ }^{1}$ This cannot be avoided in a neoplastic lesion, which may threaten the child's survival. Paediatric ovarian tumors differ from adult ovarian tumors in presentation, histology and biological behaviour and mandate a different therapeutic approach. ${ }^{3}$

MATERIALS AND METHODS: The ovarian lesions were studied over a period of 7 years at Niloufer Hospital and Institute of Child Health. Specific data collected for each patient included:

Financial or Other, Competing Interest: None.

Submission 20-10-2015, Peer Review 21-10-2015,

Acceptance 29-10-2015, Published 07-11-2015.

Corresponding Author:

Dr. K. Nagarjuna,

D. No. 4-7-12/53, Ravindra Nagar,

Nacharam, Hyderabad-500076.

E-mail: shantisrinivas@gmail.com

DOI: $10.14260 /$ jemds $/ 2015 / 2225$.
Age at presentation. Presenting symptoms and signs.

RADIOLOGICAL INVESTIGATIONS: Ultrasonogram, radiograph of abdomen and pelvis, contrast enhanced CT of abdomen and pelvis,

\author{
MRI of abdomen and pelvis Serum markers: \\ - $\alpha$ fetoprotein, $\beta$-HCG \\ - Operative reports \\ - Pathology reports.
}

RESULTS: A systematic search identified 36 patients with ovarian lesions over a 7 year period. The age at presentation ranged from newborn to 14 years. 17 patients were between 8 years and 14 years of age, 9 patients were between 1 and 8 years of age. 3 patients were under 1 year of age. 7 patients presented in the neonatal period. Neoplastic lesions were not found in neonates and infants. Both neoplastic and nonneoplastic lesions predominated in the 1 to 14 years age group. Benign neoplasms predominated in the 5 to 10 years age and malignant neoplasms were equally distributed in all age groups above 1 year of age.

The most common presenting complaint was abdominal or pelvic mass which was present in 24 cases. Second most common presenting symptom was pain abdomen which was present in 12 patients. 5 patients were detected in antenatal ultrasound examination. 4 patients presented with vomiting. 2 patients were diagnosed incidentally on ultrasound examination, which was done for some other indication. 2 cases were detected on routine physical examination and 1 patient presented with abdominal distension. 
Endocrine manifestations associated with ovarian lesions were found in 1 patient who had a follicular cyst.

Mass abdomen was the commonest symptom in both neoplastic and non-neoplastic lesions. Acute pain abdomen with fever and vomiting was the commonest presentation of torsion of ovary 4 cases of ovarian torsion were seen, out of which 3 occurred in non-neoplastic ovarian cysts and 1 occurred in a mature cystic teratoma.

Ultrasound examination revealed a cystic lesion in 18 cases, solid lesion in 5 cases and complex lesion in 13 cases. The size of the lesions was less than $5 \mathrm{~cm}$ in 10 cases, 5 to $10 \mathrm{~cm}$ in 17 cases and more than $10 \mathrm{~cm}$ in 9 cases. The most common ultrasound character of the non-neoplastic ovarian lesion was cystic whereas that of a neoplastic ovarian lesion was complex or mixed. Majority of the non-neoplastic ovarian lesions measured between 5 to $10 \mathrm{~cm}$ in size and neoplastic lesions were more than $10 \mathrm{~cm}$ in size.

Left ovarian pathology was found in 19 cases (53\%) and right ovarian pathology was found in 17 cases (47\%).

Non neoplastic lesions were present in 22 patients. Out of these 22 cases 17 had follicular cysts, 2 had luteal cysts and 3 had ovarian cyst with torsion.

Neoplastic lesions were seen in 14 patients, of which 8 were benign and 6 were malignant.

Germ cell tumors are the most common neoplastic lesions accounting for $92.8 \%$ of all tumors. There were 7 cases of mature cystic teratoma, 2 cases of dysgerminoma, 3 cases of yolk sac tumor, 1 case of malignant teratoma and 1 case of epithelial tumor. Benign serous cystadenoma was seen in 1 patient.

There were 6 cases with malignant ovarian neoplasms. $\alpha$ fetoprotein was elevated in 3 cases with yolk sac tumors and one case with malignant teratoma. Omentectomy, retroperitoneal lymphnode dissection and biopsy of contralateral ovary was avoided in children without their gross involvement. Only suspicious lymph nodes and doubtful areas were sent for biopsy. Ascetic fluid was sent for analysis, unilateral salpingo-oopherectomy was performed in all these children. Chemotherapy was given in ovarian tumors that has spread beyond the confines of the ovary.

13 cases of non-neoplastic lesions were managed by surgical interventionand 9 were managed conservatively. Conservative management was given to patients with lesions smaller than $5 \mathrm{~cm}$ in size, simple cyst, asymptomatic cyst, cysts decreasing in size on followup scan, unequivocal ovarian origin, $\alpha$ fetoprotein and $\beta$-HCG within normal limits.

DISCUSSION: Ovarian lesions are rare in childhood. ${ }^{4}$ and as a result operations for these lesions are uncommon. In Niloufer Hospital more than 3000 paediatric surgeries are performed per year and of these only 36 cases of ovarian lesions were operated in study period of 7 years. This accounts to an average of about 5 surgeries for ovarian lesions per year or $0.1 \%$ of all paediatric surgery cases. According to Cass et al series. ${ }^{5}$ from Houston, Texas which performs more than 4200 paediatric surgeries per year, there were only 7 ovarian surgeries per year which represent about $0.2 \%$ of overall paediatric surgery cases. Breen and Maxon. ${ }^{6}$ in a review of 613 cases from 10 repots found a $36 \%$ frequency of nonneoplastic lesions and $64 \%$ of neoplastic lesions. Freud et al. ${ }^{2}$ in a review of 34 girls presented an incidence of $53 \%$ for nonneoplastic and $47 \%$ for neoplastic lesions. Desilva et al reported $67.2 \%$ incidence of non-neoplastic lesions and $32.8 \%$ incidence of neoplastic lesions. In our series nonneoplastic lesions were seen in $61.11 \%$ of cases and neoplastic in $38.89 \%$ of cases. Physiological and functional ovarian lesions are the most common non-neoplastic lesions. These may occur due to failure of a follicular rupture or because the corpus luteum fails to regress. These are the commonest lesions found in our series.

Ovarian tumors are not common in children and are mostly benign in nature. ${ }^{7}$ Most paediatric ovarian neoplasms are of germ cell origin followed by sex cord stromal and epithelial tumors. In Desilva et al..$^{8}$ series $72.7 \%$ of neoplasms were of germ cell origin. Freud et al series documented $69 \%$ incidence of germ cell tumors, $19 \%$ incidence of stromal and $6 \%$ incidence of epithelial tumors. In our study the incidence of germ cell tumors is $92.8 \%$ and epithelial tumors is $7.2 \%$ there were no malignant epithelial or stromal tumors in our study. In Desilva et al series, $84 \%$ of all germ cell tumors were mature cystic teratoma.

In our study $53.8 \%$ of all germ cell tumors were mature cystic teratoma. In De silva et al study malignant tumors were rare and found only in $13.6 \%$ of cases and there was only one malignant epithelial tumor. In our study malignant neoplasms of the ovary were of germ cell origin and fortunately they account for only $16.6 \%$ of all lesions of the ovary. A fetoprotein is a sensitive marker for malignant germ cell tumors like yolk sac tumor and malignant teratoma. This tumor marker is useful in the diagnosis and follow up of these cases post operatively.

Analysis of clinical features could not distinguish between neoplastic and non-neoplastic ovarian lesions. Most common presenting complaint in paediatric ovarian lesion is abdomen or pelvic mass. Ovarian lesions may present with precocious puberty, unique to pre- menarcheal girls with hormonally active ovarian lesions. This may be caused by secretion of hormone by the lesion. It can also be seen in hormonally inactive lesions, this is due to the mass effect of the lesion causing excessive secretion. Less than $10 \%$ of precocious puberty in females is associated with ovarian lesions. Desilva et al series reported two children with nonneoplastic ovarian lesion and one child with germ cell tumors presenting with precocious puberty.

Although ovarian torsion is a rare diagnosis, it must be considered in all girls who present with lower abdominal pain, pelvic mass, nausea, vomiting and fever. ${ }^{9} 60 \%$ of ovarian torsion have underlying ovarian pathology. Most of the lesions are benign. Malignant lesions of the ovary have a lower rate of torsion due to their tendency of local invasion. The risk of torsion correlates with the size of the ovarian lesion and length of the pedicle. ${ }^{9}$ In our study 3 of the 4 ovarian torsions occurred in non-neoplastic ovarian cysts and one case of neoplastic lesion (Mature cystic teratoma) presented with torsion.

Abdominal sonography remains the most reliable diagnostic tool in the assessment of ovarian masses. In Freud et al series the appearance and size of the ovarian lesion was useful in patient management. 
In Desilva et al series cysts measuring over $5 \mathrm{~cm}$ were significant and were managed surgically. Lesions measuring more than $10 \mathrm{~cm}$ with solid or complex appearance were likely to be neoplastic, in his series the commonest cause of complex cyst was non-neoplastic lesion or haemorrhage into corpus luteal or follicular cyst. In our study the commonest appearance of non-neoplastic ovarian lesion is cystic. Majority of non-neoplastic ovarian lesions were between 5 to $10 \mathrm{~cm}$ and neoplastic lesions were over $10 \mathrm{~cm}$ in size. In our series malignant lesions were predominantly solid and benign lesions were predominantly complex.

Conservative management for non-neoplastic lesions is very important to preserve the ovarian tissue and future fertility. Even unilateral oophorectomy can impair residual ovarian function and appear to increase the risk of trisomy 21 in off spring. In Desilva et al series surgical intervention was done in $30 \%$ of children with non-neoplastic ovarian lesions. In our series surgery was performed in $59 \%$ of cases of nonneoplastic ovarian lesions.

CONCLUSIONS: Physiological or functional ovarian cysts are the commonest ovarian lesions seen in the paediatric age group. Commonest presenting complaint of paediatric ovarian lesion is abdominal or pelvic mass. Ultrasonogram is accurate and very reliable in distinguishing neoplastic from non-neoplastic and malignant from benign lesions. Nonneoplastic lesions can be managed conservatively in about 40 $\%$ of cases. Neoplastic lesions in children require a less radical approach in order to preserve ovarian function.

\section{BIBLIOGRAPHY:}

1. Mark F. Brown et al. Ovarian Masses in Children, A review of 91 cases of malignant \& benign masses. Journal of Paediatric Surgery. 1993 July; 28: p. 930-932.

2. E. Freud et al. Ovarian masses in children. Clinical Paediatrics.1999, October; 38: p. 573-577.

3. Daniel Von Allmen et al. Malignant lesions of the ovary in childhood. Seminars in Paediatric Surgery. 2005, May; 14: p.100-105.

4. Andrea Hayes-Jordan et al. Surgical management of the incidentally identified ovarian mass. Seminars in Paediatric Surgery. 2005, May; 14: p. 106-110.

5. Cass DL et al. Surgery for ovarian masses in infants, children and adolescents: 102 Consecutive patients treated in a 15 year period. J Paediatric Sur. 2001; 36: p. 693-699.

6. Breen et al. Ovarian tumors in children and adolescents. Clinical obstetrics gynaecology. 1977; 20: p. 607-623.

7. Claire L. Templeman et al. Benign Ovarian Masses. Seminars in Paediatric Surgery. 2005, May; 14: p. 93-99.

8. K.S.H. De Silva et al. Ovarian Lesions in Children and adolescence-A 11 years Review. Journal of Paediatric Endocrinology and Metabolism. 2004; 17: p. 951-957.

9. Darrell L. et al. Ovarian torsion. Seminars in Paediatric Surgery. 2005, May; 14: p. 86-92.

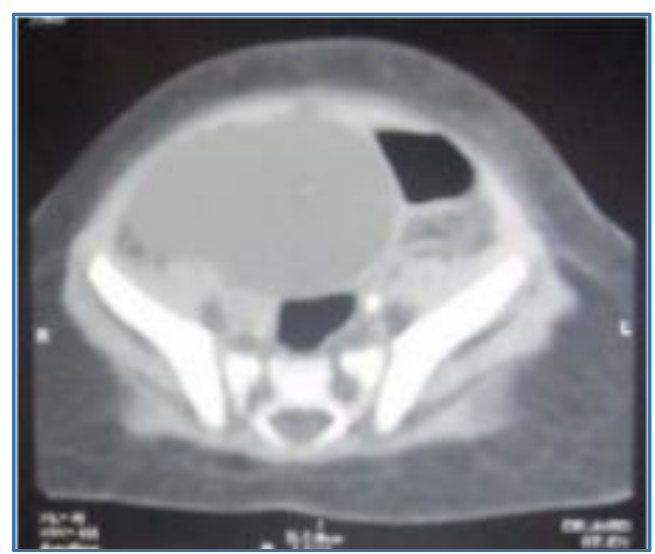

Fig. 1: Contrast enhanced CT Showing Right Ovarian Cyst

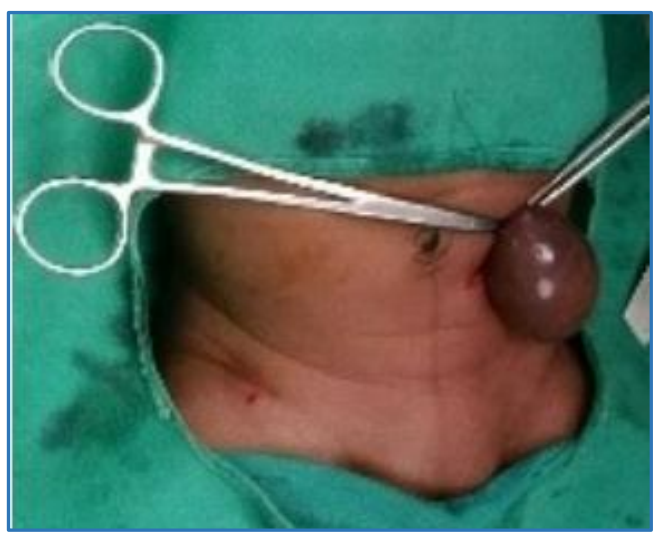

Fig. 2: Intra Operative Picture Showing Left Sided Ovarian Cyst in a Neonate 